\title{
Subsídios para o estudo das Leguminosas da Amazônia
}

\author{
William A. RODRIgues (") \\ Instituto Nacional de \\ Pesquisas da Amazônia
}

\begin{abstract}
RESUMO
A Leguminosa Peltogyne subsessilis W. Rodr. é descrita como nova espécie, e Diplotropis purpurea (Rich.) Amsh. var. leptophylla (Kl.) Amsh., D. ra cemosa (Hoehne) Amsh. var. kaieteurensis Amsh., D. ferruginea Benth. e Poecilanthe hostmanni (Benth.) Amsh. são discutidas como plantas pouco conhecidas ou novas para a Amazônia brasileira.
\end{abstract}

\section{INTRODUÇÃo}

Embora as Leguminosas da Amazônia tenham merecido especial atenção de botânicos como Ducke, por exemplo, muito ainda nos resta para conhecer inteiramente esta tão importante e útil família. A descoberta de uma nova espécie, descrita neste trabalho, é uma prova disto.

Fitogeograficamente, o reaparecimento de plantas como as mencionadas neste artigo, nas cercanias de Manaus, em pleno centro da Amazônia, antes só conhecidas das Guianas e de Minas Gerais, no Brasil, é um fato muito interessante de isolamento de espécies em duas áreas disjuntas e bastante afastadas uma da outra, sem elo conhecido até então de ligação entre elas, o que prova que nossa flora está ainda longe de ser completamente perlustrada e estudada.

\section{1 - Peltogyne Vog.}

Peltogyne subsessilis W. Rodr., n. sp. (Fig. 1)

Arbor parva vel media, cortice bunnescenti, ligno interiore brunneo mox saturate purpu- reo-violaceo, cum radiis stratosis conspicuis in sectione tangentiali; ramuli hornotini 1,0-1,5 $\mathrm{mm}$ crassi, fuscescenti, microscopice pubescenti, vetustiores crassi cortice fusco-cinereo glabrescentique; stipulis mox caducis, non vidi. Petiolus communis petiolulusque glabrus et nigrescens: ille 5,0-(6,0)-15,0 mm longus et hic $3,0-5,0 \mathrm{~mm}$ longus. Foliola $22,0-65,0 \mathrm{~mm}$ longa, $6,0-32,0 \mathrm{~mm}$ lata, falcato-oblonga ovalisve. ad basin inaequilatera et oblique-rotundata vel breve-angustata, ad apicem vulgo satis longeacuminata, discoloria et fusca, chartacea, utrimque glabra, supra minus distincte prominuli-reticulata quam infra; costa supra vix impressa, subtus salienti; nervis utrimque prominulis. Racemuli breves, pauciflori in paniculam usque ad 70,0 mm longam, modice flexuosam, terminalem et subterminalem; rachidibus tenuissimis pube brevi brunnescenti vestitis; bracteae non vidi; bracteolis ca, 2,0 mm longis, late ovatis vel suborbiculatis, concavis, brunneis, extus minute puberulis, ante anthesin caducis. Alabastrum subglobosum 2,0-2,5 $\mathrm{mm}$ diam., fulvido-sericeum, subsessile. Flores aperti eburnei, odorati; calice tubo discifero subnullo; limbi segmentis $3,0-4,0 \mathrm{~mm}$ longis, 2,5-3,0 mm latis, late ovatis, ad apicem obtusis, extus densissime fulvido-sericeis, intus glabrescentibus, glandulis insconspicuis; petalis $3,0-4,0 \mathrm{~mm}$ longis, $0,7-1,0 \mathrm{~mm}$ latis, anguste ellipticis, glandulosis; staminibus 5,0-7,0 $\mathrm{mm}$ longis, glabris. Pistillum glabrum; ovario ca. 2,0 mm longo, nigrescenti; stipite brevissimo ca. $0,3 \mathrm{~mm}$ alto, piloso; stylo ca. $4,5 \mathrm{~mm}$ longo, involuto, stigmate bifido. Legumen ca. $3,0 \mathrm{~cm}$ longum, $2,5 \mathrm{~cm}$ latum, unilateraliter dehiscens, obovato-suborbiculatum, sessile,

(") - Bolsista do Conselho Nacional de Pesquisas 

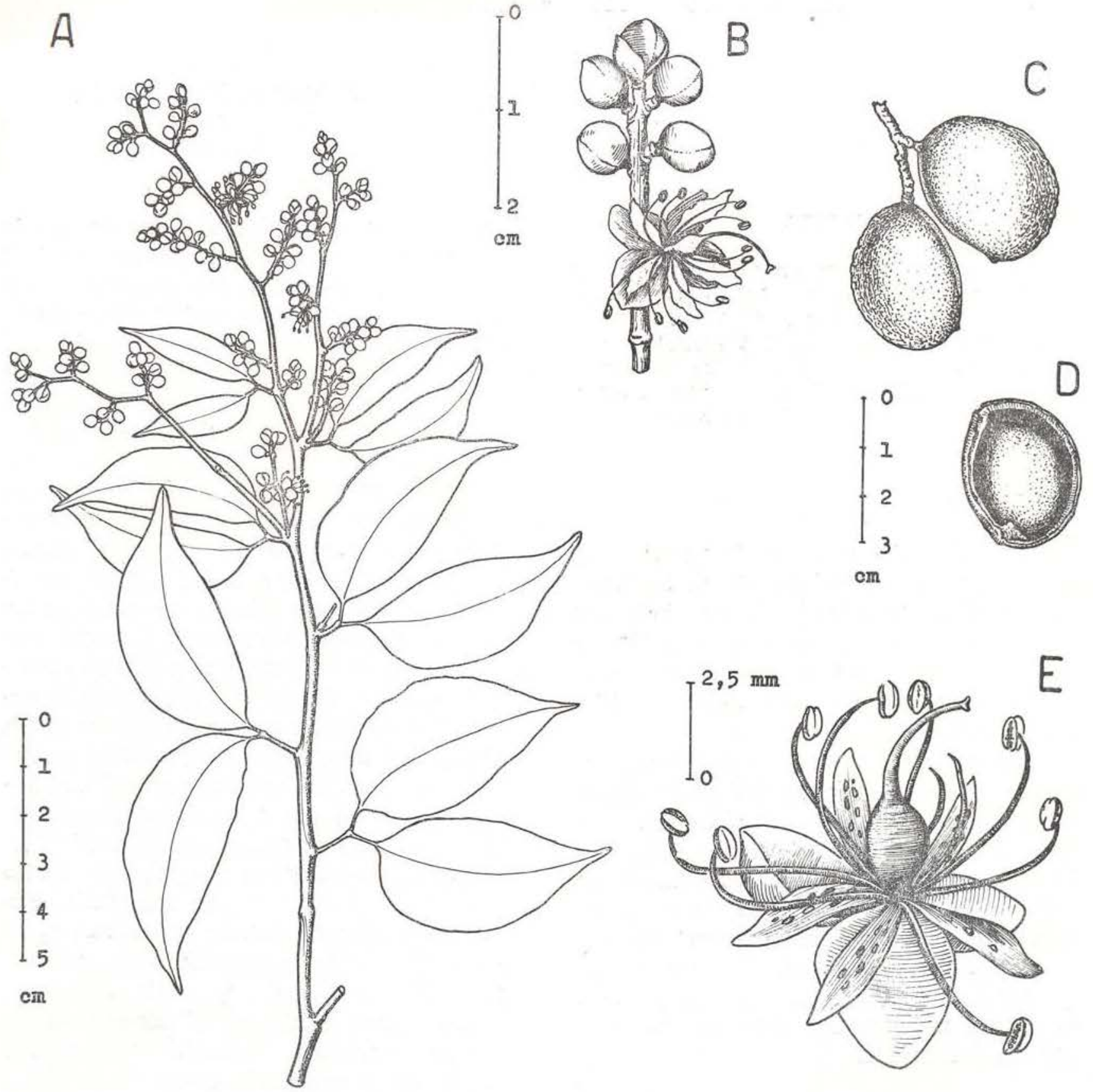

Fig. 1 - Peltogyne subsessilis W. Rodr. (L. Coelho \& D. Coelho 95). A - Ramo florífero. B - Parte da inflorescência. C - Fruto (W. Rodrigues \& A. Loureiro 7051). D - Fruto aberto mostrando uma semente. E - Flor. (Lesenhos de Júlio Dellome Filho). 
plano-convexum, crasse-coriaceum, rugulosum, in sicco fuscum vel nigricans, suturis inferioribus semicircularibus, suturis superioribus curvulis, incrassatis, non alatis. Semina 2,3-2,5 $\mathrm{cm}$ longa, $1,7 \mathrm{~cm}$ lata, elliptica, plano-convexa, testa nigrescentia, nitidula et glabra, colligulosa, arillo late cupuliformi cincta; cotyledonibus crassiusculis.

Haec species $P$. pauciflorae Benth et $P$. gracilipis Ducke proxime affinis, sed praecipue differt foliis vulgo longissime acuminatis, alabastri indumento ambitoque, floribus subsessilibus, petalis anguste ellipticis, fructu non alato et sessili, etiam quoque ligni structura in sectione tangentiali cum radiis stratosis.

Habitat in Brasilia, Amazonas, in vicinia Manaus, in silvis non inundabilibus. "Violeta" ad incolis appelata.

Typus: L. Coelho \& D. Coelho 95 (15/II/1971) in Herb. INPA 28480 (Holotypus).

Espécimes examinados:

AMAZONAS: Estrada Manaus-Itacoatiara, $\mathrm{Km}$ 64, picada I, a $3340 \mathrm{~m}$ da margem da estrada: Árvore de $16 \mathrm{~m} \times 10 \mathrm{~cm}$ de diâm., frutos verdes. W. Rodrigues \& A. Loureiro 7051 (27/VIII/1965), INPA 15713. amostra de madeira INPA X-3238; - Road ManausCaracaraí $\mathrm{Km}$ 28: Tree $17 \mathrm{~m} \times 15 \mathrm{~cm}$ diâm. Heart wood purple. "Violeta". G. T. Prance, B. S. Pena \& J. F. Ramos $3039(10 / \mathrm{XI} / 1966)$ INPA 18595, NY; Estrada Manaus-Itacoatiara $\mathrm{Km}$ 163, margem da estrada: Árvore de $20 \mathrm{~m}$ de alt. por $45 \mathrm{~cm}$ de diâm. ocasional na mata de terra firme, argilosa. Flores cremes, levemente aromáticas. L. Coelho \& D. Coelho 95 (15/II/1971) INPA 28480 (Holótipo), amostra de madeira INPA X-4180.

As espécies mais próximas de Peltogyne subsessilis W. Rodr. são $P$. pauciflora Benth. e $P$. gracilipis Ducke, distinguindo-se destas principalmente pelas folhas comumente longo acuminadas, indumento e forma dos botôes florais, flores subsésseis, pétalas elíticas estreitas, frutos não alados na sutura superior $\mathrm{e}$ sésseis.

Fato digno de menção é a tendência para a estratificação dos raiọs do lenho da espécie em questão, estrutura esta pelo menos não consignada antes por Mattos Filho $(1954,1973)$ e Metcalfe \& Chalk (1950).
2 - Diplotropis Benth.

Diplotropis purpurea (Rich.) Amsh. var. leptophylla (Kleinh.) Amsh., Meded. Bot. Mus. \& "Herb. Rijksuniv. Utrecht 52: 44.1939.

(Fig. 2)

SURINAME - Boschreserve. Sectio $O$, tree n. 608 : Forestry Bureau 1631 (fl. 14/II/1916) U.

BRASIL - Amazonas, estrada Manaus-Itacoatiara, rio Preto: Árvore de $6 \mathrm{~m}$; flores róseas, aromáticas; mata de terra firme. W. Rodrigues \& J. Cha. gas $4169(30 / \mathrm{I} / 1962)$ INPA 10734, amostra de madeira INPA X-1390; - Manaus, estrada do Tarumă, perto da estrada Manaus-Itacoatiara: Arvore de $12 \mathrm{~m}$ de alt. esgalhada desde a base por rebrotação de toco; ocasional na capoeira de terra firme, à margem da estrada; flores róseas. W. Rodrigues, D. Coelho \& O. Monteiro 8706 (12/II/1970) INPA 27878, NiG, IAN, madeira INPA X-4098; - mesma localidade e mesma árvore: W. Rodrigues 8723 (F1. 26/II/1970) INPA 27897; - mesmo local e mesma árvore: W. Rodrigues $8804(14 / \mathrm{IV} / 1970)$ fruto verde-pálido, INPA 28016, IAN.

Nova para a Amazônia brasileira. Antes só conhecida do Suriname (Amshoff, 1939). Distingue-se das demais variedades principalmente pelas nervuras secundárias subimpressas ou indistintamente elevadas na página superior, pelo reticulado obsoleto em ambas as faces e leve pubescência na página inferior Pela dimensão e pilosidade das flores é próxima à variedade brasiliensis e pelo fruto, à var. coriacea. O fruto, agora conhecido, pode ser descrito como se segue:

Legumen (ut $D$. purpurea var. coriacea Ducke) $5,0-12,0 \mathrm{~cm}$ longum, $2,0-3,5 \mathrm{~cm}$ latum subviride, membranaceum, reticulato-venosum, ad basin rotundatum et brevissime stipitatum.

As variedades brasileiras de $D$. purpurea Rich.JAmsh. podem ser reconhecidas pela seguinte chave sinóptica:

A) Folíolos subcoriáceos; flores ferrugíneas ou canotomentosas

a) Foliolos com reticulado distinto em ambas as faces; flores ferrugíneas 1 - brasiliensis (Tul.)Amsh. 


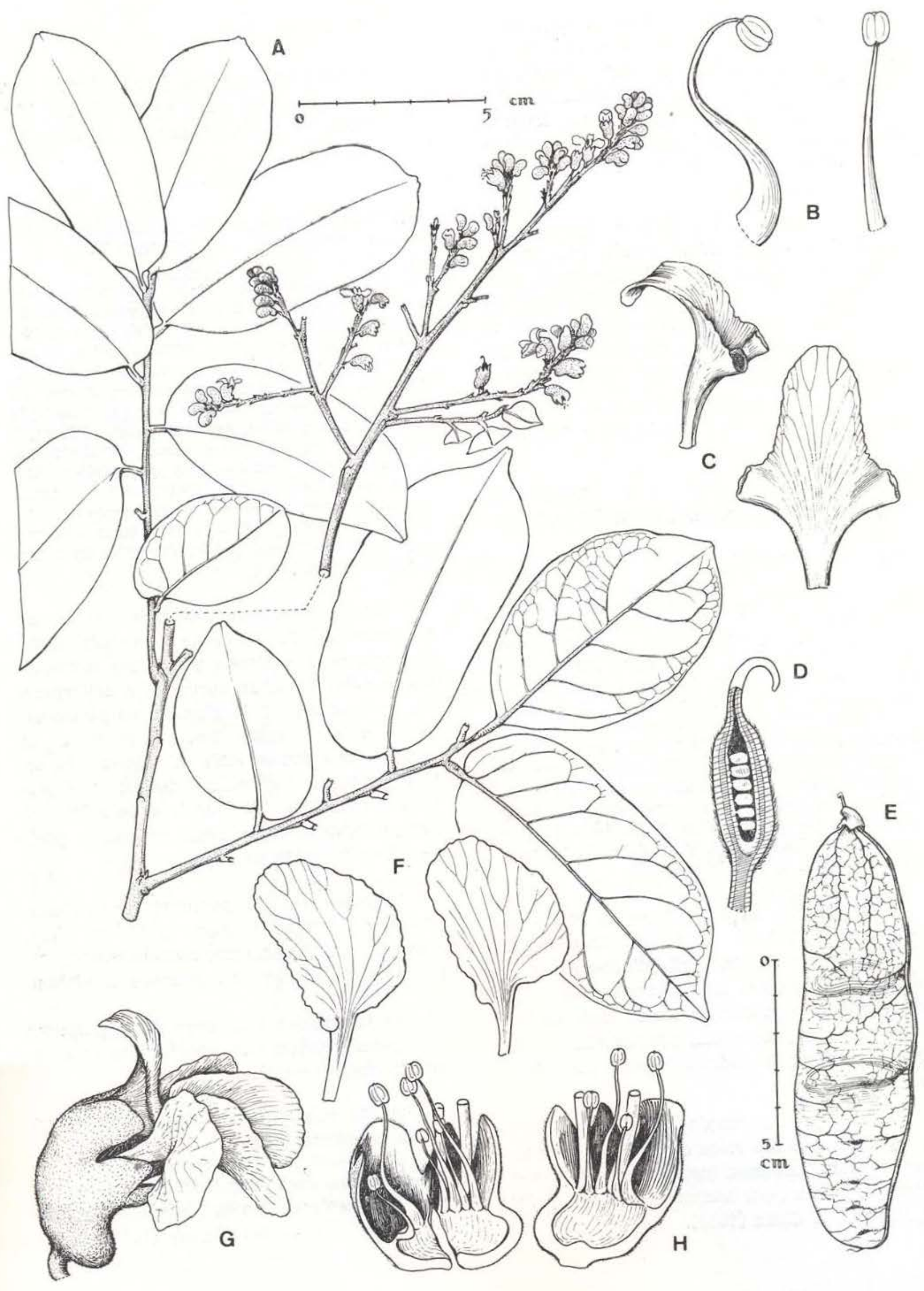


b) Foliolos com reticulado distinto na face superior e obsoleto na inferior; inflo. rescência canotometosa.

\section{2 - belemnensis Ducke}

c) Folíolos com reticulado obsoleto ou quase indistinto em ambas as faces e leve pubescência na página inferior; inflorescência ferrugínea

$$
3 \text { - leptophylla (Kleinh.)Amsh. }
$$

B) Folíolos coriáceos; flores rufo-pubescente escuras e um tanto maiores que nas demais variedades

$$
4 \text { - coriacea Ducke }
$$

Diplotropis racemosa (Hoehne)Amsh var. kaieteurensis Amshoff Bull. Torrey Bot. Club 75 (4):393. 1948.

(Fig. 3)

BRASIL - Amazonas, Manaus, Ponte da Bolívia: Arvoreta de $6 \mathrm{~m} \times 12 \mathrm{~cm}$ de diâm., ocasional na capoeira, solo silicoso úmido, perto de um córrego; frutos esverdeados. W. Rodrigues 8627 (12/XII/1969) INPA 27739, amostra de madeira INPA X-4132; - mesmo local e mesma planta: W. Rodrigues 8668, flores róseas (27/I/1970) INPA 27840, MG; - mesmo local: Árvore de $10 \mathrm{~m} \times 15$ $\mathrm{cm}$ de diâm., flores lilazes. W. Rodrigues 8760 (10/III/1970) INPA 27934.

Primeira ocorrência desta variedade no Brasil. Antes só conhecida da Guiana no mesmo tipo de solo e em vegetação aparentemente semelhante fisionomicamente. Diferencia-se da espécie principalmente pelas folhas menores e mais largas, de cor pardo-escuras depois de herborizadas e pelo fruto rígido-coriáceo, indistintamente reticulado.

Diplotropis ferruginea Benth., Mart. FI. Bras. 15(1):321. 1859.

BRASIL - Amazonas, estrada Manaus-Itacoatiara

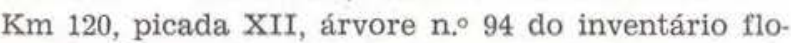
restal: Árvore de $22 \mathrm{~m} \times 29 \mathrm{~cm} \mathrm{D}$. A. P.; mata de terra firme; nome vern.: "Sucupira preta". W. Ro. drigues 8040 (st. 22/IX/1965) INPA 17400, amostra de madeira INPA X-3642; - ibid., Km 64, Reserva W. Egler: Árvore grande com $45 \mathrm{~cm}$ de diâm., ocasional na mata de terra firme; frutificação abundante; frutos verde-pálidos colhidos do chão, em fase de germinação. W. Rodrigues, D. Coelho \& $\mathbf{O}$. Monteiro 9077 (5/VI/1973) INPA 38332.

Espécie rara em Minas Gerais ou pelo menos muito poucas vezes recoletada, a sua redescoberta na Amazônia, pela primeira vez aqui assinalada, amplia bastante a sua área de distribuição.

Comparado o nosso espécime frutífero com o exemplar florífero de Capanema (RB $n$. 5049) colhido em São Gonçalo do Rio das Pedras (Minas Gerais), ambos são muito semeIhantes na parte vegetativa, não só quanto à pilosidade dos ramos e folhas como quanto à consistência e número de folíolos (5 a 9). 0 fruto maduro, até então desconhecido, pode agora ser descrito:

Legumen ut D. purpurea (Rich.)Amsh., $12,0-14,5 \mathrm{~cm}$ longum, 3,5-4,0 cm latum, oblongum, indescens, membranaceum, glabrum, reti venularum utrimque prominente, 1- vel 2-spermum; semen ca. $3,0 \mathrm{~cm}$ longum, $0,5 \mathrm{~cm}$ latum, molle, exalbuminosum, planum.

A germinação da semente não tem período de dormência, germina logo ao tocar o solo. O epicótilo é densamente revistido de tomento amarelado.

\section{3 - Poecilanthe Benth.}

Poecilanthe hostmanni (Benth.)Amshoff, Meded. Bot. Mus. \& Herb. Rijksuniv. Utrecht 52: 61. 1939.

BRASIL - Amazonas, estrada Manaus-Itacoatiara, $\mathrm{Km} 155$, no varadouro para a picada XIX, lado direito: Árvore de $12 \mathrm{~m}$ de alt. x $15 \mathrm{~cm}$ de diâm., frequente na mata de terra firme, solo argiloso, tronco irregular com pequena sapopema; frutos verdes, brilhosos. W. Rodrigues 9001 (11/II/1971) INPA

Fig. 2 - Diplotropis purpurea (Rich.)Amsh. var. leptophylla (Kleinh.)Amsh. (W. Rodrigues \& al. 8706. A Ramo florífero. B - estames, C - Pétada vexilar vista frontal e de lado. D - Pistilo seccionado longitudinal mente. E - Fruto. (W Rodrigues 880). F - Pétala alada e carinada, G - Flor inteira. H - Cálice seccionado mostrando os estames. (Desenhos de Júlio Dellome Filho). 


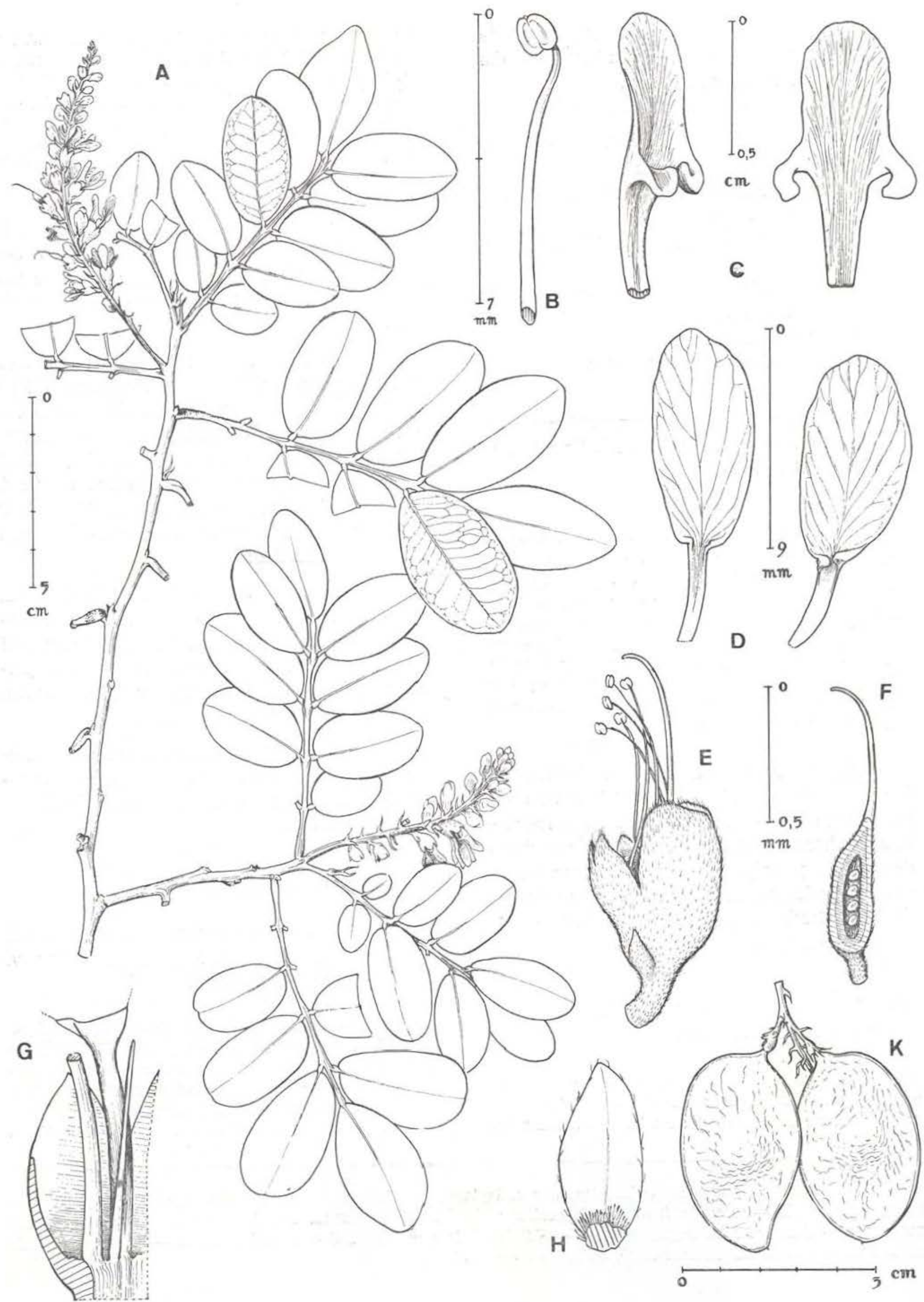


28471, amostra de madeira INPA X-4178 com leve

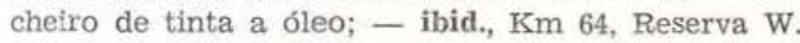
Egler: Arvore de $8 \mathrm{~m} \mathrm{x} 15 \mathrm{~cm} \mathrm{D.} \mathrm{A.} \mathrm{P.,} \mathrm{frequiente}$ localmente na mata de terra firme; base do tronco com raízes aéreas; ramiflora; flores com cálice verde-claro e corola purpúrea. W. Rodrigues, D. Coelho \& O. Monteiro 9076 (5/VI/1973) INPA 38331.

Nova para o Estado do Amazonas. Sua distribuição anterior se limitava à Guiana, Suriname e ao Oiapoque, no Território brasileiro do Amapá (Ducke, 1959), próximo à Guiana Francesa.

Planta freqüente nos locais onde foram observadas. Quando jovem tem folhas pinadas até 7 folíolos opostos ou subopostos, grandes.

\section{SUMMARY}

In this paper the Leguminous Peltogyne subcessilis W. Rodr. is described as new species for Amazonia, and Diplotropis purpurea (Rich.) var. leptophylla (Kl.) Amsh., D. racemosa (Hoehne)
Amsh, var. kaieteurensis Amsh., D. ferruginea Benth. and Poecilanthe hostmanni (Benth.) Amsh, are discussed here as interesting woody plants little known or new to brazilian Amazonia.

\section{BIBLIOGRAFIA CITADA}

AMSHOFF, G. JANE H.

1939 - On the South American Papilionaceae. Meded. Bot. Mus. \& Herb. Rijksuniv., Utrecht, 52:1-78.

DUCKE, A.

1959 - Notas adicionais às Leguminosas da Amazônia brasileira. Bol. Téen. IAN, Belém, 36:65.

Mattos Filho, A.

1954 - Anatomia do lenho de Peltogyne Vog. Arq. Serv. Flor., Rio de Janeiro, 8:45-154.

1973 - Anatomia do lenho de Peltogyne recifensis Ducke. Arq. Jard. Bot. Rio de Janeiro, 19:125-131.

Metcalfe, C. R. \& Chalk, L.

1950 - Anatomy of the Dicotyledons. Oxford, Claredon Press. 2 v.

Fig. 3 - Diplotropis racemosa (Hoehne) Amsh. var. kaieteurensis Amsh. (W. Rodrigues 8668). A - Ramo florífero. B - Estame. C - Pétala vexilar vista de lado e de frente. D - Pétala alada e carinada. E - Flor sem a corola. F - Pistilo seccionado. G - Cálice parcialmente seccionado mostrando a inserção de estames e pétala. H - Bracteola. K - Frutos. (W. Rodrigues, 8627). (Desenhos de Júlio Dellome Filho). 